\title{
Plasma Exchange as an Adjunctive Therapy for Crescentic IgA Nephropathy
}

\author{
Xinfang Xie Jicheng Lv Sufang Shi Li Zhu Lijun Liu Min Chen Yu Wang \\ Zhao Cui Xin Wang Li Liu Xiaojuan Yu Fude Zhou Ming-hui Zhao \\ Hong Zhang
}

Renal Division, Peking University First Hospital, Peking University Institute of Nephrology, Key Laboratory of Renal Disease, Ministry of Health of China, and Key Laboratory of Chronic Kidney Disease Prevention and Treatment, Peking University, Ministry of Education, Beijing, China

\section{Key Words \\ Crescentic IgA nephropathy · Plasma exchange . \\ Intensive immunosuppressive treatment $\cdot$ Renal failure . \\ Propensity score matching $\cdot$ Cohort study}

\begin{abstract}
Background: Crescentic IgA nephropathy (CrelgAN) has a poor prognosis despite aggressive immunosuppressive therapy. The efficacy of plasma exchange (PE) in CrelgAN is not well defined. Methods: Twelve patients with severe CrelgAN who received $\mathrm{PE}$ as addition to routine immunosuppressive therapy, followed for more than 6 months, were involved. Twelve matched historical controls who received immunosuppressive therapy alone were selected by propensity score matching. Renal survival, plasma IgA-lgG complex and active complement products were assessed. Results: Nine men and 3 women received a median of 7 PE courses (range 5-10). Their baseline urine protein excretion rate was $5.8(4.5-8.7) \mathrm{g} /$ day, and their serum creatinine level was $705.3 \pm 296.4 \mu \mathrm{mol} / \mathrm{l}$. During a mean follow-up of 15.6 months (6-51 months), 6 of the 12 PE group patients were free of dialysis, while all the control patients were dialysis dependent ( 6 of 12 vs. 0 of 12, p=0.014). In the PE group, dialysis had to be restarted for 1 patient owing to the devel-
\end{abstract}

opment of severe pneumonia and pulmonary failure. PE was associated with a higher kidney survival rate (log rank test, $\mathrm{p}=0.026$ ) during follow-up. It also significantly decreased plasma IgA-IgG complex levels (pre-PE: $85.3 \pm 25.9 \%$ vs. postPE: $38.4 \pm 12.4 \%, p<0.001$ ) and plasma and urinary active complement product levels, including $\mathrm{C} 3 \mathrm{a}, \mathrm{C} 5 \mathrm{a}$ and soluble $\mathrm{C} 5 \mathrm{~b}-9$. The latter levels remained low until the last follow-up. Conclusion: This study indicated that PE could increase renal recovery rates in severe CrelgAN.

(c) 2016 S. Karger AG, Basel

\section{Introduction}

IgA nephropathy (IgAN) is one of the most common glomerulonephritis and is characterized by a highly variable clinical course and diverse histopathological lesions [1]. Although most affected individuals develop chronic slowly progressive renal injury, a subgroup of patients ( $<5 \%$ of all IgAN patients) have diffuse crescent formation, which is termed as crescentic IgAN (CreIgAN) and often leads to rapidly progressive kidney failure. The recent Kidney Disease: Improving Global Outcomes (KDIGO) guidelines suggest high-dose steroids and cyclophosphamide therapy for CreIgAN [2]. However, this suggestion is

\section{KARGER}

E-Mail karger@karger.com

www.karger.com/ajn
(C) 2016 S. Karger AG, Basel

0250-8095/16/0442-0141\$39.50/0
Jicheng Lv or Hong Zhang

Renal Division, Peking University First Hospital

No. 8, Xishiku Street

Xicheng District, Beijing 100034 (China)

E-Mail jichenglv75@gmail.com or hongzh@bjmu.edu.cn 
mainly based on several small observational studies [3-5], and the 1- and 5-year renal survival rates of patients treated with this regimen were as low as $65 \%$ and $28 \%$, respectively, in one large cohort of CreIgAN patients [6].

Plasma exchange (PE) therapy has been shown to be beneficial in crescentic glomerulonephritis caused by anti-neutrophil cytoplasmic antibody (ANCA)-associated vasculitis or anti-glomerular basement membrane (GBM) antibody disease [7, 8]. However, there is only poor-quality evidence to support the use of PE in CreIgAN. One anecdotal report indicated a benefit of $\mathrm{PE}$ in combination with immunosuppressive therapies in 5 IgAN patients [9]. Furthermore, this has been confirmed in other case reports [10-13].

In our prior study including 113 CreIgAN patients, we found that patients with severe CreIgAN and serum creatinine (SCr) $>600 \mu \mathrm{mol} / \mathrm{l}$ were unlikely to recover from chronic dialysis, even when combined with aggressive immunosuppressive therapy [6]. We have therefore added $\mathrm{PE}$ therapy to the initial treatment regimen for severe CreIgAN in our clinical practice since 2011. In this study, we aim to determine the long-term efficacy and safety of the addition of $\mathrm{PE}$ in the initial treatment regimen of patients with severe CreIgAN.

\section{Methods}

\section{Patients}

CreIgAN patients who were referred to the renal division of Peking University First Hospital and received PE treatment were enrolled in this study. In a previous cohort study, we observed that CreIgAN patients do not recover after they reach a stage requiring dialysis [6]. Therefore, from November 2011, we have considered adding PE treatment within 2 weeks after kidney biopsy in patients with diffuse active crescent formation and rapidly progressive kidney failure, who also met at least one of the following criteria: (1) $\mathrm{SCr}>600 \mu \mathrm{mol} / \mathrm{l},(2)$ requirement of dialysis and (3) persistent deterioration of kidney function even after treatment with high-dose steroids and other immunosuppressive agents. Patients with serum ANCA or anti-GBM autoantibodies were excluded from this study. We selected matched controls from a cohort of 63 CreIgAN patients by performing propensity score matching based on initial $\mathrm{SCr}$ and pulse methylprednisolone, oral steroid and immunosuppressant treatments.

Patients were followed up for at least 6 months or until they progressed to end-stage kidney disease (ESKD) and were dependent on dialysis for at least 6 months.

\section{Treatments, Follow-Up and Outcome Measures}

Plasmapheresis was carried out every other day or every 3 days, and we used 2-3 liters plasma volumes replacement fluid with 5\% albumin or fresh frozen plasma in each session. In all PE procedures, low molecular weight heparin/heparin was used for anticoagulants and single vascular access was used. Immunosuppressive therapy was administered as part of the routine intervention strategy in our clinical practice, provided there were no contraindications to it, such as active infection or fibrosis of most crescents. The aggressive immunosuppression regimen included pulse methylprednisolone (7-15 mg/kg/day for 3 days) followed by oral prednisone $(0.8-1 \mathrm{mg} /$ $\mathrm{kg} /$ day; maximum dose, $60 \mathrm{mg} /$ day) combined with other immunosuppressive agents, such as cyclophosphamide $(2 \mathrm{mg} / \mathrm{kg} /$ day $)$ or mycophenolate mofetil (1-2 g/day). The therapeutic regimen was adjusted according to the renal function and histological lesions.

The primary outcomes of interest were renal function recovery and ESKD or death. Renal function recovery was defined as independent of dialysis or SCr $<200 \mu \mathrm{mol} / \mathrm{l}$ within 6 months and lasts without a first relapse until at least 12 months. ESKD was defined as patients who had received chronic dialysis for at least 6 months or undergone renal transplantation.

\section{Plasma IgA1 and IgA-IgG Complex}

Blood samples were collected at Peking University First Hospital with the informed consent of the study participants. All protocols were reviewed and approved by the Institutional Review Boards at the Peking University First Hospital. Plasma from 8 patients was collected before and after PE treatment and at the last follow-up. The plasma samples were aliquoted and stored at $-80^{\circ} \mathrm{C}$ until analysis. In 2 patients, only pre-PE and last follow-up samples were available. Plasma circulating immune complexes were isolated using polyethylene glycol precipitation, as previously described [14]. The final protein concentration was determined using BCA Protein Assay Kit (Pierce Biotechnology, USA). IgA-IgG complexes were measured using ELISA. Microtitration plates were coated with $10 \mu \mathrm{g} / \mathrm{ml} \mathrm{F}(\mathrm{ab}$ ') 2 fragment of goat anti-human IgA (for crosscapture of IgA-IgG complexes, Jackson Immuno-Research Labs, West Grove, Pa., USA), as described. Precipitated plasma (1:400 dilution) was then added and revealed with horseradish peroxidase-labeled rabbit anti-human IgG (1:40,000 dilution, Sigma-Aldrich). The plates were developed with the peroxidase chromogenic substrate o-phenylenediamine $-\mathrm{H}_{2} \mathrm{O}_{2}$, and the reaction was stopped with $1 \mathrm{M}$ sulfuric acid. The absorbance was measured at $450 / 570 \mathrm{~nm}$. The immune complex levels were expressed as the ratio of the optical density of the sample to that of the positive control.

\section{Plasma and Urine Complement Component Levels and Urine}

Interleukin-6

Urine from only 5 patients was collected before PE treatment and at the last follow-up. The samples were aliquoted and stored at $-80^{\circ} \mathrm{C}$. Plasma and urine concentrations of human complement components (including C5a, C3a and soluble C5b-9 (sC5b-9)) were determined using ELISA (Quidel, San Diego, Calif., USA). All the complement components were assayed according to the manufacturer's instructions. ELISAs for the measurement of the urinary concentration of interleukin (IL)- 6 were obtained from R\&D systems (USA).

\section{Statistical Analyses}

Normally distributed quantitative variables and non-normally distributed variables were expressed as means and SDs, and as medians and interquartile ranges, respectively. Categorical data were summarized as ratios and percentages. Differences in the means of continuous variables among the pre-PE, post-PE and last followup samples were compared using the paired samples $t$ test or non- 
Fig. 1. Treatment regimens of consecutive patients with CreIgAN. Response to treatment indicates that the patient did not have deterioration of renal function or showed renal function improvement after treatment in hospitalization.

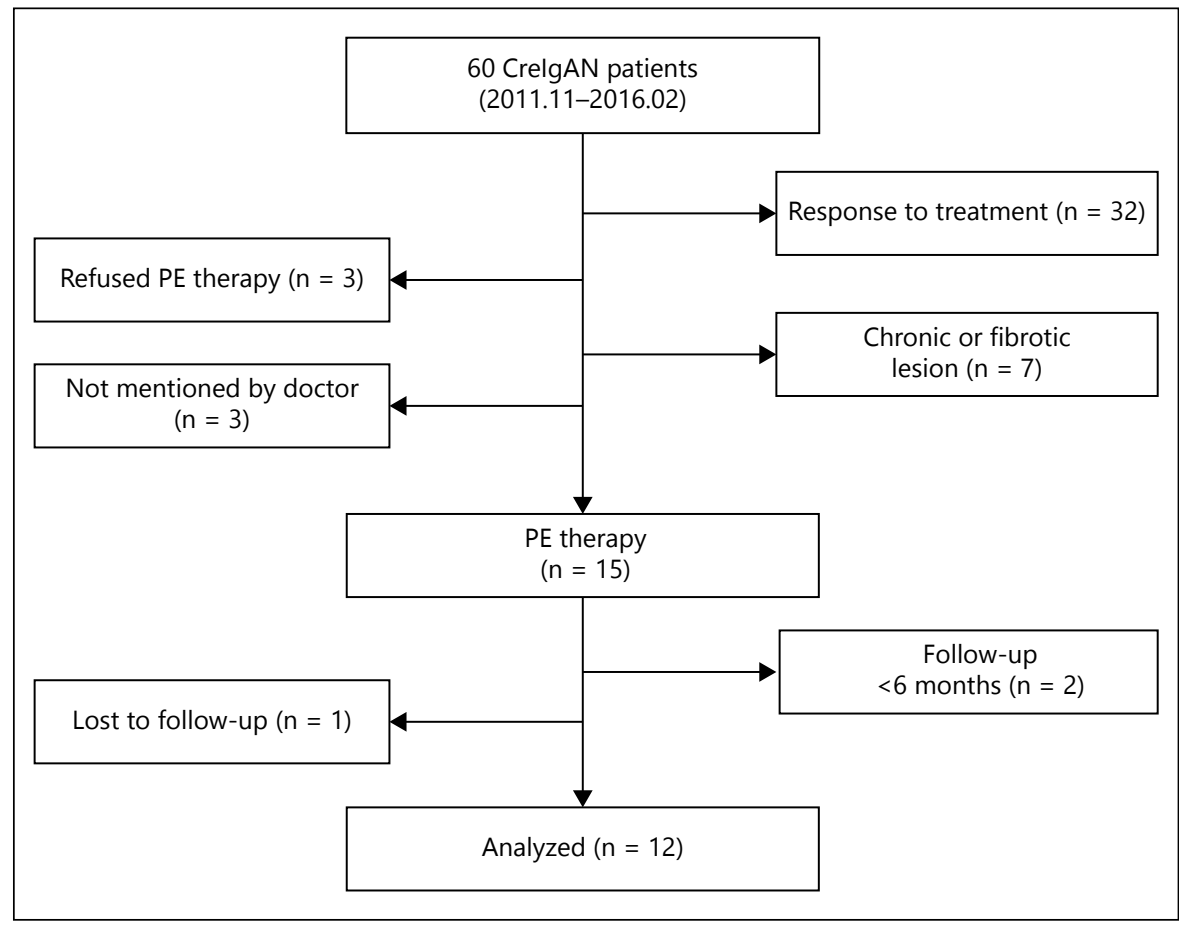

parametric tests for 2-related samples, that is, the Wilcoxon signed-rank test. A 2 -sided $\mathrm{p}$ value $<0.05$ was regarded as statistically significant. Statistical analyses were performed using Stata 13.0 and SPSS 16.0.

\section{Results}

\section{Baseline Characteristics}

From November 2011 to February 2016, 15 consecutive patients with evidence of diffuse crescent formation and rapidly progressive kidney failure received $\mathrm{PE}$ as an additional therapy. Ten of these patients required dialysis, while the other 5 did not require dialysis but exhibited kidney function deterioration even after aggressive immunosuppressive therapy. Of the 15 patients, 12 were followed up for at least 6 months and were included in this analysis (fig. 1). Three patients were not included in the analysis, 1 patient who received only 3 courses of PE was lost to follow-up and the other 2 with improved kidney function after PE were followed up 2 and 4 months each. Twelve historical controls who were matched for initial SCr, steroids and immunosuppressants therapy and without PE were selected from a cohort of 63 CreIgAN patients by propensity score matching. The baseline characteristics of the 2 groups are shown in table 1 . The PE group contained 9 men and 3 women with a mean age of $42.7 \pm 15.0$ years; their baseline urine protein excretion rate was $5.8(4.5-8.7) \mathrm{g} /$ day, and their SCr level was $705.3 \pm 296.4 \mu \mathrm{mol} / \mathrm{l}$. Eight of these patients had nephrotic syndrome. Eleven of the patients had hypertension. Several other complications were also present in this group: 1 patient had severe pulmonary infection and a history of stroke and coronary heart disease, 1 had active tuberculosis, 1 had obsolete pulmonary tuberculosis, 1 had hepatitis B virus infection and 1 had bronchiectasis.

The mean percentage of crescents was $64.4 \pm 24.4 \%$ (the percentages of cellular, fibrocellular and fibrous crescents were $29.8 \pm 17.1,34.7 \pm 20.9$ and $0 \%(0-22.2 \%)$, respectively), and 6 patients had more than $50 \%$ tubular atrophy and interstitial fibrosis lesion in the PE group. There was no significant difference in the baseline clinical characteristics or histological lesions between the study patients and the matched historical controls (online suppl. table S1; for all online suppl. material, see www.karger. com/doi/10.1159/000448767).

\section{Treatments and Follow-Up}

$\mathrm{PE}$ treatment was initiated at a median of 9 days (range 4-27 days) after kidney biopsy. A mean of 7 PE sessions was performed per patient (range 5-10 sessions per patient) during a median period of 15 days (range 9-30 days). Nine patients each in the PE and control groups were treated with oral steroids and immunosuppressive agents (table 2). Nine patients in each group received intravenous 
Table 1. Baseline characteristics of patients in the PE and control groups

\begin{tabular}{|c|c|c|}
\hline & PE group $(n=12)$ & Control group $(n=12)$ \\
\hline Age, years, mean $\pm S D$ & $42.7(15.0)$ & $40.8(15.5)$ \\
\hline Sex, male & 9 & 11 \\
\hline History, months, median (IQR) & $1.5(1.0-5.0)$ & $5.5(1.3-78.0)$ \\
\hline Macrohematuria & 5 & 3 \\
\hline Hypertension & 11 & 12 \\
\hline $\mathrm{SBP}, \mathrm{mm} \mathrm{Hg}$, mean $\pm \mathrm{SD}$ & $148.3(15.2)$ & $152.5(18.3)$ \\
\hline $\mathrm{DBP}, \mathrm{mm} \mathrm{Hg}$, mean $\pm \mathrm{SD}$ & $92.8(13.1)$ & $90.4(13.9)$ \\
\hline RPGN/ARF & 11 & 11 \\
\hline $\mathrm{SCr}, \mu \mathrm{mol} / \mathrm{l}$, mean $\pm \mathrm{SD}$ & $705.3(296.4)$ & $661.7(249.4)$ \\
\hline Oliguria & 2 & 2 \\
\hline Proteinuria, g/day, median (IQR) & $5.8(4.5-8.7)$ & $4.7(4.0-5.8)$ \\
\hline Proteinuria $>3.5 \mathrm{~g} /$ day & 8 & 10 \\
\hline Albumin, $g / l$, mean $\pm S D$ & $26.6(3.3)$ & $29.6(4.5)$ \\
\hline Dialysis at presentation & 8 & 9 \\
\hline \multicolumn{3}{|l|}{ Pathology } \\
\hline Total glomeruli & $21(7)$ & $20(8)$ \\
\hline Total crescent, $\%$, mean \pm SD & $64.4(24.4)$ & $69.5(25.5)$ \\
\hline Cellular crescent, $\%$, mean \pm SD & $29.8(17.1)$ & $31.4(16.8)$ \\
\hline Fibrocellular crescent, $\%$, mean \pm SD & $34.7(20.9)$ & $33.4(18.8)$ \\
\hline Fibrous crescent, \%, median (IQR) & $0(0-22.2)$ & $11.8(1.9-20.3)$ \\
\hline Global glomerulosclerosis, $\%$, mean $\pm \mathrm{SD}$ & $4.6(8.5)$ & $6.2(5.6)$ \\
\hline \multicolumn{3}{|l|}{ Tubular atrophy/interstitial fibrosis, n (\%) } \\
\hline $1-50$ & $6(50)$ & $4(33.3)$ \\
\hline$>50$ & $6(50)$ & $8(66.7)$ \\
\hline
\end{tabular}

RPGN = Rapidly progressive glomerulonephritis; ARF = acute renal failure.

Table 2. Treatments and clinical outcomes of patients in the PE and control groups

\begin{tabular}{lcc}
\hline & PE group (n=12) & Control group (n=12) \\
\hline Follow-up, months, mean \pm SD & $15.6(14.8)$ & $7.9(5.3)$ \\
Courses of PE/patient, median (range) & $7(5-10)$ & 0 \\
Initial PE from kidney biopsy, days, median (range) & $9(4-27)$ & - \\
Duration of PE treatment, days, median (range) & $15(9-30)$ & - \\
Replacement fluid liters/course, ml, mean \pm SD & $2,517(300)$ & - \\
Pulse methylprednisolone & 9 & 9 \\
Steroids + immunosuppressants & 9 & 9 \\
Steroids alone & 3 & 3 \\
Cyclophosphamide & 9 & 7 \\
Mycophenolate mofetil & 0 & 2 \\
RAS blocker & 5 & 5 \\
Free of dialysis after treatment & $6 *$ & 0 \\
ESKD & 7 & 12 \\
Death & 1 & 3 \\
\hline
\end{tabular}

Immunosuppressive agents included cyclophosphamide, mycophenolate mofetil and leflunomide. * For 1 patient, dialysis had to be restarted owing to the development of severe pneumonia and pulmonary failure 1 month later. 


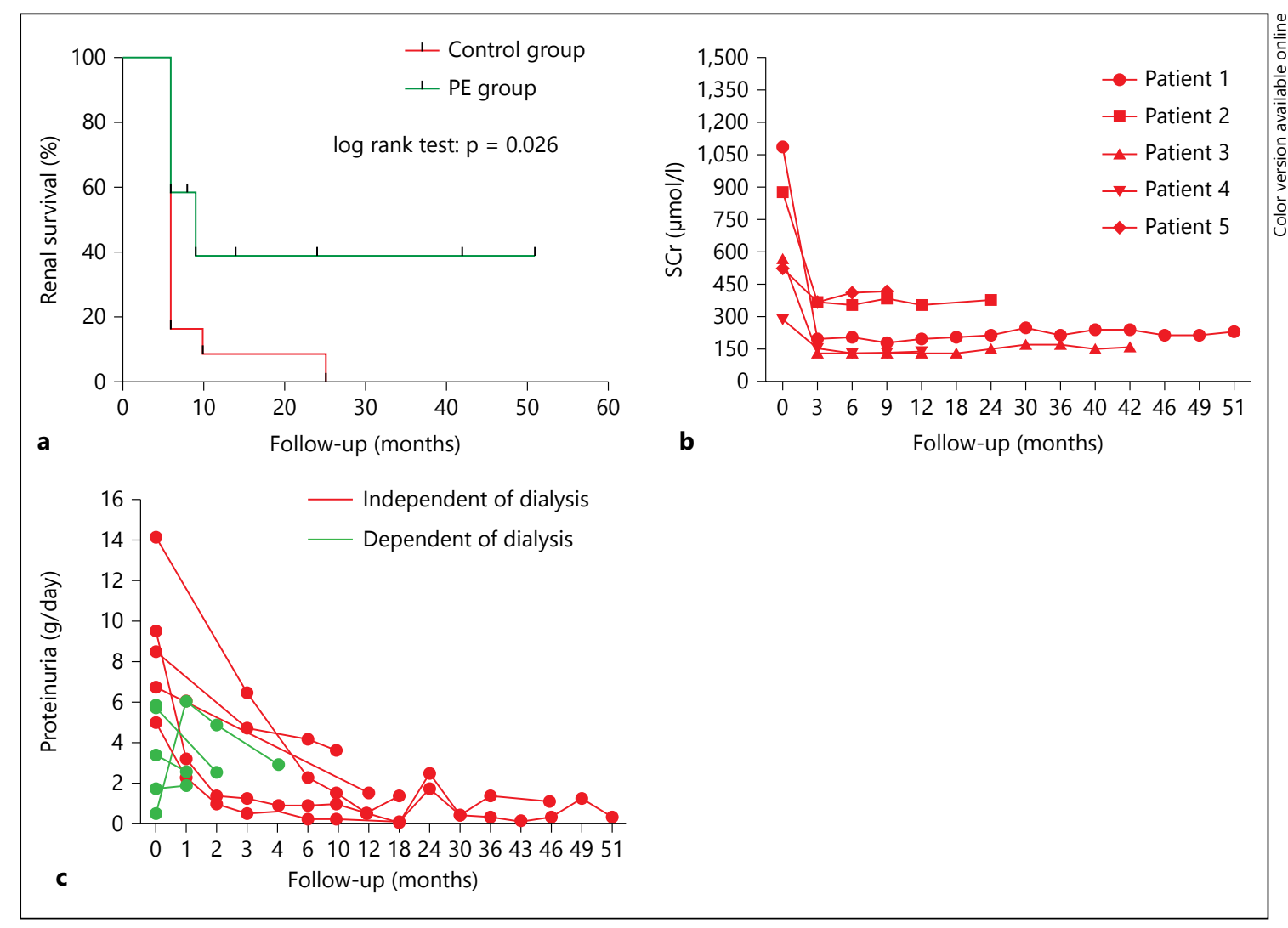

Fig. 2. a-c Renal survival in patients receiving PE therapy, and the SCr levels and degree of proteinuria during follow-up.

pulse methylprednisolone, and 3 patients in each group received only oral steroids without immunosuppressive agents. Three patients in the PE group did not receive intravenous methylprednisolone owing to pulmonary infection or acute heart failure and stroke history. There was no significant difference in drug therapy between the PE group and control group (online suppl. table S1).

In the PE group, 4 of the 8 patients who were dependent on dialysis successfully recovered within 4 months (range 1 week to 4 months) after the kidney biopsy. However, in 1 patient, the dialysis had to be restarted owing to the development of severe pneumonia and pulmonary failure 1 month later. In contrast, none of the 9 dialysis patients in the control group recovered. Overall, after a mean follow-up duration of 15.6 months (range 6-51 months), 6 of the 12 patients in the PE group were free of dialysis, while all the patients in the control group were dialysis dependent ( 6 of 12 vs. 0 of $12, p=0.014$ ). PE therapy was associated with a higher kidney survival rate (log rank test, $\mathrm{p}=0.026$; fig. $2 \mathrm{a}$ ), and the patients who were free of dialysis had stable SCr levels and proteinuria re- duction throughout the follow-up, while for those with dialysis dependence, regular proteinuria measurements were not available because of oliguria or anuria during follow-up (fig. 2b, c).

\section{Plasma IgA-IgG Complex and Complement Fragment} Levels

We examined the profiles of plasma IgA-IgG complex and complement activation products before $\mathrm{PE}$, after $\mathrm{PE}$ and at the last follow-up in patients for whom sequential samples were available. There was a significant decrease in plasma IgA-IgG complex level after PE therapy (prePE: $85.3 \pm 25.9 \%$ vs. post-PE: $38.4 \pm 12.4 \%, \mathrm{p}<0.001$ ) and a partial reversal to the baseline levels by the last followup (70.6 $\pm 24.6 \%$; fig. 3a). PE treatment also significantly reduced the levels of plasma C3a, C5a and sC5b-9 (fig. 3bd), all of which remained low until the last follow-up. Patients who were independent of dialysis had lower plasma complement activation products levels at the last followup than those who remained dependent on dialysis, although the difference was not significant (online suppl. 


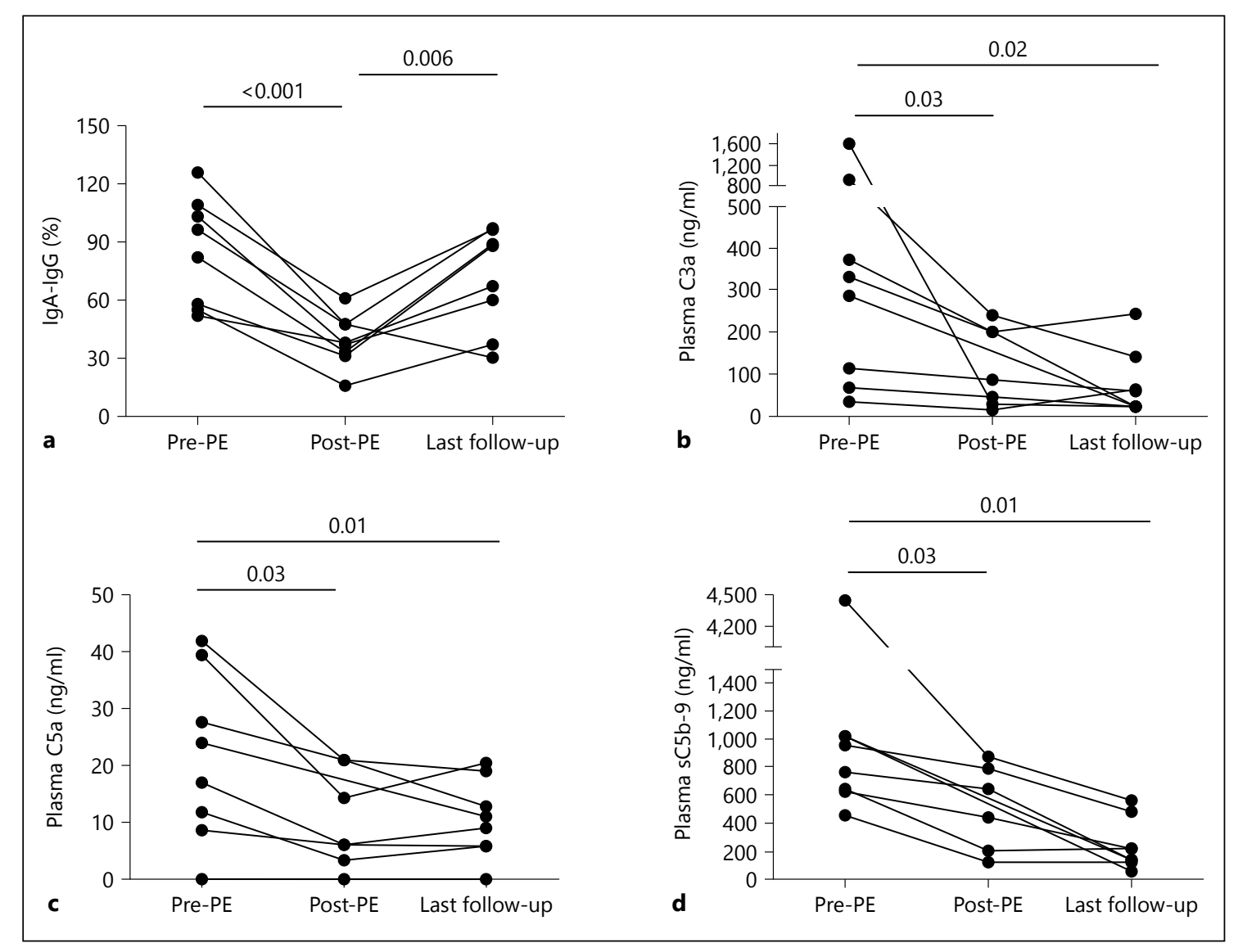

Fig. 3. a-d Change of IgA1, IgA-IgG complexes and complement activation products in plasma after PE therapy. The numbers over the black bars represent $\mathrm{p}$ values.

fig. S1). Similar to the plasma levels, the urinary levels of C3a, C5a and sC5b-9 in 4 patients (with urine samples available) significantly decreased after PE treatment and remained low until the last follow-up. The changes in urinary IL-6 level showed the same trend (fig. 4).

\section{Safety and Adverse Events}

$\mathrm{PE}$ therapy in addition to immunosuppressive agents was well tolerated by most patients. One patient with a history of bronchiectasis developed severe pneumonia and pulmonary failure 1 month after discharge.

\section{Discussion}

Severe CreIgAN has poor outcomes, especially in those requiring dialysis at presentation, as these patients rarely recover from dialysis. In this study, we studied the data of 24 patients with severe CreIgAN (mean SCr $>650$ $\mu \mathrm{mol} / \mathrm{l})$. The control group patients who were treated with aggressive immunosuppressive therapy progressed to ESKD during follow-up. In contrast, in the PE group, 4 of the 8 patients who received dialysis recovered, among whom 1 restarted dialysis owing to the development of severe pneumonia and pulmonary failure 1 month later; 2 of the other 4 patients had progressive CreIgAN but did not need dialysis while hospitalization and had stable renal function with SCr fluctuates in 150-250 $\mu \mathrm{mol} / \mathrm{l}$ and 360-420 $\mu \mathrm{mol} / \mathrm{l}$ after PE therapy. The others remained dependent on dialysis or progressed to dialysis until the last follow-up. In addition, PE treatment reduced the levels of IgA-IgG complex and plasma complement activation products, which have been suggested to be involved in the pathogenesis of IgAN progression $[15,16]$. Thus, this study indicated that the addition of PE to immunosuppressive therapy increases the renal recovery rate in severe CreIgAN patients.

The importance of this study is that it provides a new treatment alternative for patients with severe CreIgAN requiring dialysis. The $2012 \mathrm{KDIGO}$ guidelines suggest the 


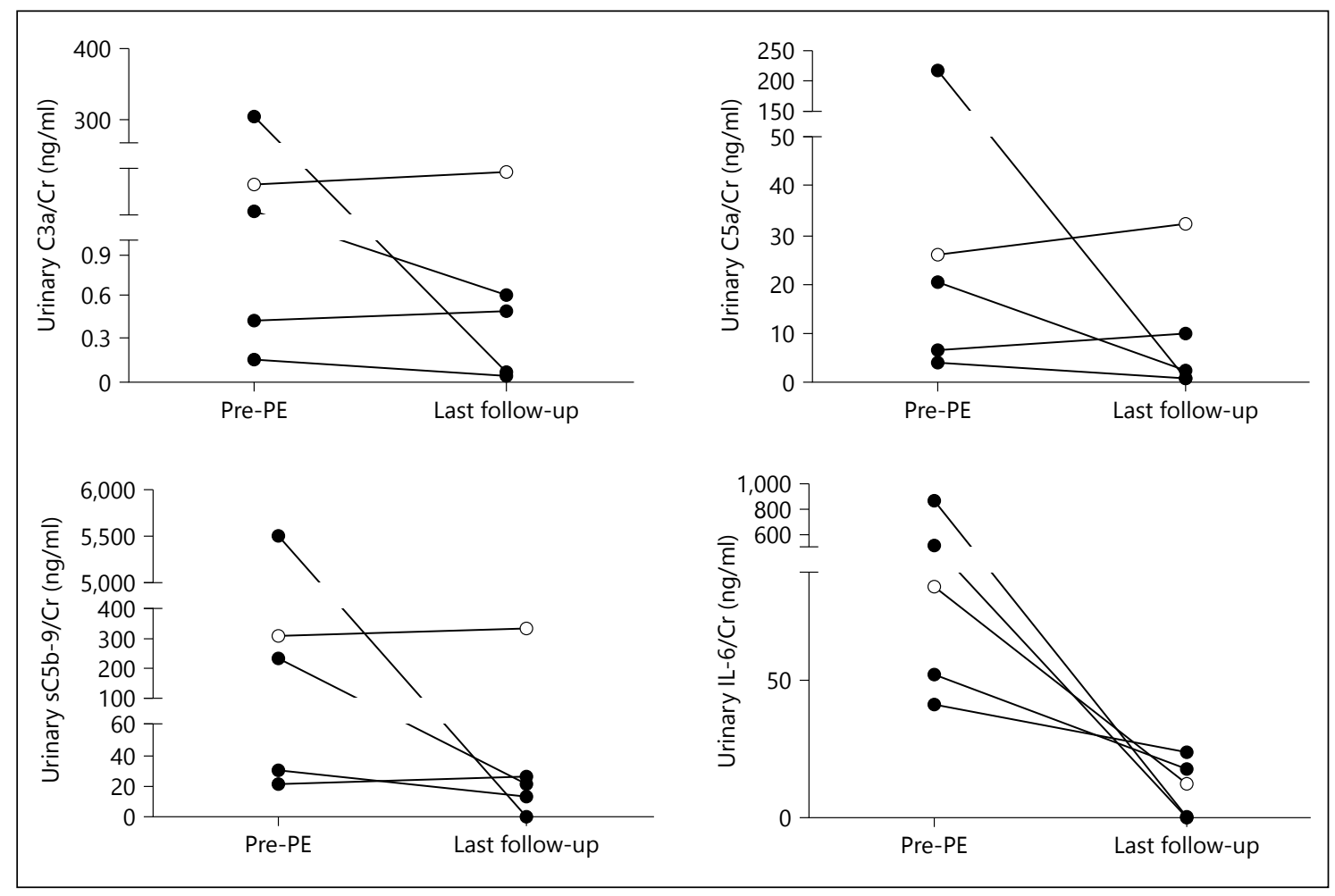

Fig. 4. Change of complement activation products and IL-6 in urine after PE therapy. Solid circles were the levels of patients independent of dialysis during the follow-up, the hollow was the level of patients dependent on dialysis during the follow-up.

use of steroids and cyclophosphamide in patients with rapidly progressive CreIgAN [2]. However, this recommendation was mainly based on small observational studies, and has not been evaluated in large populations. In our prior study with 113 CreIgAN patients, we found low 3- and 5 -year kidney survival rates of $46 \%$ and $30 \%$, respectively. Furthermore, among the patients with severe CreIgAN (SCr level $>600 \mu \mathrm{mol} / \mathrm{l}$ ), only $3 \%$ were able to achieve independence from dialysis during follow-up [1]. Our present study has indicated a new optional treatment for this patient population. Case reports and case series from previous decades have addressed the PE treatment of IgAN patients with rapidly progressive kidney failure and crescent formation on renal biopsy (online suppl. table S2) [913, 17-21]. Coppo et al. [9] studied the effects of PE in 5 patients with progressive IgAN and found that PE delayed disease progression in the 3 patients with crescent formation, but did not delay progression in the patients with advanced sclerotic lesions [13]. In another 2 reports, PE treatment successfully restored kidney function in a child with CreIgAN and rapid renal function deterioration despite intravenous methylprednisolone therapy $[10,22]$. How- ever, this benefit has not been observed in other reports [21], while some reports have found that PE treatment only slowed renal function deterioration and delayed the timeto-dialysis dependence $[11,13,17,20]$. PE has been shown to benefit kidney function in adults [19] and children [18] with severe Henoch-Schönlein purpura. Recently, PE and eculizumab in addition to intensive immunosuppressive therapy were used to treat a 16-year-old boy with HenochSchönlein purpura and CreIgAN, the treatment remarkably improved the renal function of this patient, but gradual progression to ESKD occurred after about 1 year [23]. Only one controlled trial has been performed on the PE treatment of CreIgAN patients [17], it included 3 patients treated with corticosteroids and immunosuppressants and 6 patients who also received PE. Two of the 3 patients who received only immunosuppressive therapy became dialysis dependent, while the 6 patients receiving PE demonstrated resolution of renal failure during the therapy [17]. Our study thus represents the largest controlled study evaluating the efficacy of PE in severe CreIgAN. We included 12 patients with severe CreIgAN and 12 matched controls, and most of our patients required acute dialysis. Our find- 
ings indicate that the addition of PE treatment to routine immunosuppressive therapy would significantly reduce the risk of kidney failure and increase the chances of recovering from dialysis.

The pathogenesis of IgAN is not well defined, but experimental and clinical data have established the crucial role of aberrantly glycosylated IgA 1 and anti-glycan antibodies to the abnormal IgA1 in the pathogenesis of IgAN $[15,16]$. The levels of circulating galactose-deficient IgA1 (Gd-IgA1) and anti-glycan antibodies are closely associated with kidney disease progression [24-26]. The formation of both Gd-IgA1 and anti-glycan antibody complexes activates the complement pathway, stimulates mesangial cells and induces cytokine secretion, resulting in inflammation and fibrosis $[15,27-30]$. The urinary levels of complement activation products were much higher in our patients than in patients with other IgAN (median C3a/urinary creatinine $(\mathrm{Cr})$ : CreIgAN, $14.0 \mathrm{ng} / \mathrm{mg}$ vs. other IgAN, $0.02 \mathrm{ng} / \mathrm{mg}$; median C5a/urinary Cr: CreIgAN, $30.4 \mathrm{ng} / \mathrm{mg}$ vs. other IgAN, $0.4 \mathrm{ng} / \mathrm{mg}$; median Sc5b-9/urinary Cr: CreIgAN, $311.4 \mathrm{ng} / \mathrm{mg}$ vs. other IgAN, $1.34 \mathrm{ng} / \mathrm{mg}$; all $\mathrm{p}$ values $<0.01$; online suppl. fig. S2). Furthermore, we observed that PE treatment transiently decreased the circulating levels of IgA-IgG complexes. PE treatment also significantly reduced the plasma and urine levels of complement activation split products, which remained low throughout the follow-up. In addition, the levels of the inflammatory cytokine IL- 6 decreased after $\mathrm{PE}$ therapy. This partially explains the mechanism underlying the effects of PE treatment in CreIgAN. However, serum IgA-IgG complex only transiently decreased and increased subsequently and thus PE treatment may only retard the crescents forming in the acute phase and its long-term effect is still unknown (fig. 3).

The strength of this research is that it represents the largest study of PE treatment in CreIgAN, a rare phenotype of IgAN. Furthermore, unlike other case series, this study included comparable controls selected using propensity score matching. The major limitation of this study is its retrospective study design and small sample size. Also this is a single-center study, and we cannot exclude selection bias as, especially in the control group, all of them progressed to end-stage of kidney disease even with an intensive immunosuppressive therapy. In this study, we mainly recruit patients with $\mathrm{SCr}>600 \mu \mathrm{mol} / \mathrm{l}$, which represents a much more severe condition. In fact, results from a multicenter study showed that patients hardly recovered from dialysis in such a condition. A high-quality, multicenter, specifically powered trial involving CreIgAN patients is required to evaluate the efficacy of PE. Such a trial is being planned by our study group (the RESCUE study: NCT02647255).

In conclusion, this retrospective study found that patients with severe CreIgAN treated with the addition of PE showed benefit effect compared to patients with intensive immunosuppressive therapy alone. The PE therapy decreased the circulating levels of IgA-IgG complexes, and also decreased the levels of complementary activation split products. Furthermore, PE treatment significantly reduced the risk of kidney failure and increased the chances of recovering from dialysis. Therefore, $\mathrm{PE}$ should be considered in patients with severe CreIgAN. However, given the small sample size and retrospective design of our study, we cannot draw a definite conclusion from this study. A high-quality, randomized controlled trial is still needed.

\section{Acknowledgments}

This work was supported by grants from the National Natural Science Foundation of China (Nos. 81270795) and Excellent Young Scientists Fund of the National Natural Science Foundation of China (No. 81322009), the Major State Basic Research Development Program of China 973 Program grant (No. 2012CB517700), the National Key Technology R\&D Program (No. 2013BAI09B14), the Program for New Century Excellent Talents in University from the Ministry of Education of China grant NCET-12-0011 and the Natural Science Fund of China to the Innovation Research Group (No. 81321064).

\section{Disclosure Statement}

All authors declared no competing interests. The results presented in this paper have not been published previously in whole or part, except in abstract format. Part of this study (clinical outcomes of 10/12 cases in PE group) were presented in abstract form at the American Society of Nephrology, San Diego, Calif., USA, November 3-8, 2015.

\section{References}

1 D'Amico G: Natural history of idiopathic IgA nephropathy and factors predictive of disease outcome. Semin Nephrol 2004;24 179-196.

2 Kidney Disease: Improving Global Outcomes (KDIGO) Glomerulonephritis Work Group: KDIGO Clinical Practice Guideline for Glomerulonephritis. Kidney Int Suppl 2012;2: 139-274.

3 Abe T, Kida H, Yoshimura M, et al: Participation of extracapillary lesions (ECL) in progression of IgA nephropathy. Clin Nephrol 1986;25:37-41.

4 Tang Z, Wu Y, Wang QW, et al: Idiopathic IgA nephropathy with diffuse crescent formation. Am J Nephrol 2002;22:480-486.
Xie/Lv/Shi/Zhu/Liu/Chen/Wang/Cui/ Wang/Liu/Yu/Zhou/Zhao/Zhang 
5 Pankhurst T, Lepenies J, Nightingale P, Howie AJ, Adu D, Harper L: Vasculitic IgA nephropathy: prognosis and outcome. Nephron Clin Pract 2009;112:c16-c24.

$6 \mathrm{Lv}$, Yang Y, Zhang H, et al: Prediction of outcomes in crescentic IgA nephropathy in a multicenter cohort study. J Am Soc Nephrol 2013;24:2118-2125.

7 Jayne DR, Gaskin G, Rasmussen N, et al: Randomized trial of plasma exchange or highdosage methylprednisolone as adjunctive therapy for severe renal vasculitis. J Am Soc Nephrol 2007;18:2180-2188.

8 Levy JB, Turner AN, Rees AJ, Pusey CD: Long-term outcome of anti-glomerular basement membrane antibody disease treated with plasma exchange and immunosuppression. Ann Intern Med 2001;134:10331042.

9 Coppo R, Basolo B, Roccatello D, et al: Immunological monitoring of plasma exchange in primary IgA nephropathy. Artif Organs 1985; 9:351-360.

10 Fujinaga S, Ohtomo Y, Umino D, et al: Plasma exchange combined with immunosuppressive treatment in a child with rapidly progressive IgA nephropathy. Pediatr Nephrol 2007; 22:899-902.

11 Nicholls K, Becker G, Walker R, Wright C, Kincaid-Smith P: Plasma exchange in progressive IgA nephropathy. J Clin Apher 1990; 5:128-132.

12 Tsunoda Y: [Effect of plasma exchange therapy on IgA nephropathy in children - a retrospective study]. Nihon Jinzo Gakkai Shi 1995; 37:238-246.

13 Lai KN, Lai FM, Leung AC, Ho CP, Vallance-Owen J: Plasma exchange in patients with rapidly progressive idiopathic IgA nephropathy: a report of two cases and review of literature. Am J Kidney Dis 1987;10:6670.

14 Tissandié E, Morelle W, Berthelot L, et al: Both IgA nephropathy and alcoholic cirrhosis feature abnormally glycosylated IgA 1 and soluble CD89-IgA and IgG-IgA complexes: common mechanisms for distinct diseases. Kidney Int 2011;80:1352-1363.

15 Suzuki H, Kiryluk K, Novak J, et al: The pathophysiology of IgA nephropathy. J Am Soc Nephrol 2011;22:1795-1803.

16 Wyatt RJ, Julian BA: IgA nephropathy. N Engl J Med 2013;368:2402-2414.

17 Roccatello D, Ferro M, Coppo R, Giraudo G, Quattrocchio G, Piccoli G: Report on intensive treatment of extracapillary glomerulonephritis with focus on crescentic IgA nephropathy. Nephrol Dial Transplant 1995;10:20542059.

18 Shenoy M, Ognjanovic MV, Coulthard MG: Treating severe Henoch-Schönlein and IgA nephritis with plasmapheresis alone. Pediatr Nephrol 2007;22:1167-1171.

19 Augusto JF, Sayegh J, Delapierre L, et al: Addition of plasma exchange to glucocorticosteroids for the treatment of severe HenochSchönlein purpura in adults: a case series. Am J Kidney Dis 2012;59:663-669.

20 Chambers ME, McDonald BR, Hall FW, Rabetoy GM: Plasmapheresis for crescentic IgA nephropathy: a report of two cases and review of the literature. J Clin Apher 1999;14:185187.

21 Nicholls K, Walker RG, Dowling JP, KincaidSmith P: 'Malignant' IgA nephropathy. Am J Kidney Dis 1985;5:42-46.
22 Yamazaki Y, Mori A, Nomura Y, et al: [A case of rapidly progressive IgA nephropathy with transient hypocomplementemia at onset]. $\mathrm{Ni}$ hon Jinzo Gakkai Shi 1997;39:765-770.

23 Ring T, Pedersen BB, Salkus G, Goodship TH: Use of eculizumab in crescentic IgA nephropathy: proof of principle and conundrum? Clin Kidney J 2015;8:489-491.

24 Zhao N, Hou P, Lv J, et al: The level of galactose-deficient IgA1 in the sera of patients with IgA nephropathy is associated with disease progression. Kidney Int 2012;82:790-796.

25 Berthoux F, Suzuki H, Thibaudin L, et al: Autoantibodies targeting galactose-deficient IgA1 associate with progression of IgA nephropathy. J Am Soc Nephrol 2012;23:15791587.

26 Suzuki Y, Matsuzaki K, Suzuki H, et al: Serum levels of galactose-deficient immunoglobulin (Ig) A1 and related immune complex are associated with disease activity of IgA nephropathy. Clin Exp Nephrol 2014;18:770-777.

27 Maillard N, Wyatt RJ, Julian BA, et al: Current understanding of the role of complement in IgA nephropathy. J Am Soc Nephrol 2015;26: 1503-1512.

28 Wyatt RJ, Kanayama Y, Julian BA, et al: Complement activation in IgA nephropathy. Kidney Int 1987;31:1019-1023.

29 Roos A, Bouwman LH, van Gijlswijk-Janssen DJ, Faber-Krol MC, Stahl GL, Daha MR: Human IgA activates the complement system via the mannan-binding lectin pathway. J Immunol 2001;167:2861-2868.

30 Hiemstra PS, Gorter A, Stuurman ME, Van Es LA, Daha MR: Activation of the alternative pathway of complement by human serum IgA. Eur J Immunol 1987;17:321-326. 